\title{
Cuidado nutricional em hospitais públicos de quatro estados brasileiros: contribuições da avaliação em saúde à vigilância sanitária de serviços
}

\author{
Nutritional care in public hospitals of four Brazilian states: \\ contributions of health evaluation to health surveillance services
}

M arismary H orsth DeSeta ${ }^{1}$

Gisele O'D wyer ${ }^{1}$

PatríciaH enriques ${ }^{2}$

Gizene Luciana Pereira de Sales ${ }^{3}$

${ }^{1}$ Departamento de

Administração e

Planejamento em Saúde,

Escola Nacional deSaúde

Pública Sergio Arouca,

Fundação Oswaldo Cruz.

Rua Leopoldo Bulhões

1.480 , sala 728 ,

M anguinhos. 21041-210

Rio deJaneiro RJ.

deseta@ensp.fiocruz.br

${ }^{2}$ Departamento de Nutrição

Social, Faculdadede

Nutrição, Universidade

Federal Fluminense.

${ }^{3}$ Faculdade deN utrição,

Universidade Potiguar.
Abstract Considering the importance of nutritional care for the quality of care provided to hospitalized patients, the study aims to assess health care in eight public hospitals that have received support from QualiSUS in four Brazilian states. There were semi-structured interviews with nutritionists, direct observation and review of 219 records of patients admitted to the medical clinics. The findings suggest weaknesses in the hospital nutritional care, poor integration between the production of food and nutritional care in the surveyed hospitals, in addition to structural deficiencies. We highlight problems related to the nutritional care process, including its documentation. In $93 \%$ of therecords therewereno records on the nutritional status on admission, or nutritional assessment of patients in nutritional therapy. It was observed that the assessment made by the health surveillance in routine inspections, focusing on the verification of compliance and structural aspects, does not include the detection of a possible nutritional risk for the patient. We suggest the inclusion of other criteria and strategies for surveillance, among them a review of open medical records.

Key words Nutritional care, Health evaluation, Review of medical records, Health surveillance, Inspection, Hospitals
Resumo Considerando a importância do cuidado nutricional para a qualidade da atenção prestada ao paciente internado, a pesquisa objetivou avaliar esse cuidado em oito hospitais públicos que receberam aportes do QualiSUS em quatro estados do país. Realizaram-se entrevistas semiestruturadas com nutricionistas, observação direta e revisão de 219 prontuários de pacientes internados nas clínicas médicas. Os achados sugerem fragilidades no cuidado nutricional hospitalar; deficiente integração entre produção de refeições e assistência nutricional nos hospitais pesquisados, além de deficiências estruturais. Ressaltam-se problemasrelacionados ao processo de cuidado nutricional, inclusive na sua documentação. Em 93\% dos prontuários não havia registros do estado nutricional na admissão, nem deavaliação nutricional dos pacientes em terapia nutricional. A ponta-se que a avaliação realizada pela vigilância sanitária em inspeções de rotina, centrada na verificação do cumprimento de normas e nos aspectos estruturais, não contempla a detecção deum possível risco nutricional para o paciente. Sugere-se a inclusão de outros critérios e estratégias pela vigilância sanitária, dentre elesa revisão de prontuários abertos.

Palavras-chave Cuidado nutricional, Avaliação em saúde, Revisão de prontuários, Vigilância sanitária, Inspeção, H ospitais 
Introdução

Os serviços de alimentação e nutrição institucionais orientam-se principalmente para a oferta de refeições nutricionalmente equilibradas e seguras do ponto de vista da qualidade higiênico-sanitária e, adicionalmente, para a recuperação ou manutenção da saúde dos indivíduos ${ }^{1}$. Essas orientações principais são plenamente compreensíveis pela importância da alimentação equilibrada e o envolvimento dos serviços de alimentação na ocorrência de grande parte dos surtos de origem alimentar².

Quando a instituição é o hospital, que tem como objetivo principal a recuperação da saúde, a atenção integral ao paciente sob internação abrange um conjunto de cuidados, dentre estes os relacionados à alimentação e à nutrição em seus diferentesníveis de complexidade e de intervenção, de acordo com as características individuais e o tipo de enfermidade. Com eles, além da oferta de alimentos seguros, se objetiva: corrigir e evitar deficiências nutricionais que concorrem para o aumento das complicações e da mortalidade; identificar em tempo hábil os pacientesque requerem um apoio nutricional especializado e individual e, ao reconhecer os benefícios de se alimentarem os pacientes hospitalizados com seus alimentos favoritos, oferecer um leque de dietas que contribua para amenizar o sofrimento da doença e da internação ${ }^{3}$. Esses cuidados, chamados genericamente de assistência ou cuidado nutricional, incluem avaliação do estado nutricional do paciente, identificação de metas terapêuticas, escolha das intervenções a serem implementadas, identificação das orientações necessárias ao paciente e formulação de um plano de avaliação, devidamente documentado ${ }^{4}$.

O cuidado nutricional no ambiente hospitalar tem sua centralidade na equipe de nutrição e pressupõe forte cooperação das várias categorias profissionais - médicos, enfermeiros, farmacêuticos, entre outros profissionais da equipe de saúde - no intuito de que se alcancem os resultados almejados ${ }^{5}$. Ademais, o termo cuidado re mete a modos de organização do conhecimento que ultrapassam fronteiras tradicionais de divisão em disciplinas fechadas ${ }^{6}$ e por categorias profissionais. Para tanto, além dos conhecimentos sobre al imentos e nutrição, torna-se imprescindível que os profissionais tenham clareza de seus papéis e responsabilidades no processo do cuidado nutricional ${ }^{6}$. Como exemplos, que o nutricionista assuma o desafio da efetividade da sua intervenção, inclusive no campo da educação nutricional, que provoque mudanças nos hábitos alimentares dos indivíduos e de suas famíli$\mathrm{as}^{7}$, e que a equipe de enfermagem e os médicos revalorizem o cuidado nutricional no contexto mais amplo do cuidado integral ao paciente ${ }^{6}$.

Considerada a centralidade da equipe de nutrição no cuidado nutricional, afirma-se quegrande parte do processo desse cuidado se apoia em duas subunidades que contam com distintas estruturas físicas e funcionais. Uma é responsável pelo atendimento clínico-nutricional; e a outra, pelo planejamento, produção e distribuição de refeições. A qualidade e a função da alimentação hospitalar dependem da interação entre a produção de refeições ea assistência nutricional propriamente dita, enten dida como atividade multiprofissional complexa. Essa interação éessencial para contribuir para a superação de uma fragmentação da assistência que tem atingido o trabal ho de diversas categorias profissionais da área da saúde, refletindo-senos diversos processos de cuidado. Ao se analisarem os currículos dos cursos de nutrição - eaqui se afirma que isso não é privativo da graduação em nutrição -, verifica-seque há autores que os apontam como moldados sob a vertente biológica da atenção clínico-assistencial estruturada no modelo biomédico dominante ${ }^{7}, 0$ que contribui para essa fragmentação.

A literatura nacional einternacional tem ressaltado o impacto da hospitalização no estado nutricional; a importância epidemiológica da desnutrição hospitalar em pacientes internados, sejam eles adultos em clínicas específicas, sejam idosos ou crianças, e o suporte nutricional. Para a realidade brasileira, os dados do Inquérito Brasileiro deAvaliação Nutricional Hospitalar (I branutri), de 1999, apontaram uma elevada prevalência de desnutrição nos hospitais atribuída às precárias condições alimentares anteriores à internação e/ou à deterioração do estado nutricional após ela $a^{8}$ O estudo revelou que $48,12 \%$ dos doentes internados na rede pública do país encontravam-se desnutridos e que a desnutrição hospitalar progride na medida em que aumenta o período de internação ${ }^{9}$. N essa mesma direção, 0 estudo de Garcia et al. ${ }^{10}$ verificou que, durante a internação, $29 \%$ dos pacientes perderam peso. E M ello et al. ${ }^{11}$ mostraram que em $30 \%$ a $50 \%$ dos pacientes internados pode ser diagnosticado algum grau de desnutrição.

0 reconhecimento dessa questão como relevante no cuidado do paciente, em especial no contexto brasileiro, não parece ter gerado as mudanças necessárias no âmbito hospitalar. Desse modo, a questão permanece como um pro- 
blema que deve ser estudado e enfrentado no bojo das ações de atenção nutricional ${ }^{3}$. De outra parte, há uma multiplicidade de métodos para avaliação e intervenção nutricional desenvolvidos para dar conta de diferentes contextos do cuidado e de características específicas de pacientes ou grupos de pacientes.

Este estudo não objetivou identificar, avaliar ou recomendar um método ou protocolo específico, mas nele se assumequeénecessário aprimorar a atenção prestada ao paciente internado e que, para isso, é fundamental o aprimoramento de processos específicos - em pauta, o referente ao cuidado nutricional. Assim, alguns padrões referentes a esse cuidado têm sido estabelecidos para avaliar a qualidade do cuidado em saúde, tanto nacional quanto internacionalmente.

A ação de Estado no campo da saúde no Brasil para minimizar ou eliminar riscos à saúde re lacionados com a alimentação hospitalar é levada a cabo pela vigilância sanitária. Para averiguar a propriedade dos alimentos para o consumo humano hospitalar e as condições sanitárias dos estabelecimentos enelas intervir, a vigilância sanitária real iza inspeções em hospitais e demanda análise de produtos coletados nos laboratórios oficiais. Essas inspeções são calcadas em roteiros que, no caso da alimentação hospitalar, privilegiam componentes de estrutura dos setores de produção de alimentos e os produtos utilizados, em detrimento da avaliação mais abrangente da qualidade do processo de cuidado nutricional. Dentre os aspectos considerados, podese citar a qualidade dos insumos utilizados para suporte nutricional. Dos não considerados, a verificação da realização ou não, pelo hospital, da avaliação e do acompanhamento do estado nutricional dos pacientes, mormente dos queestão sob risco nutricional. Ou seja, a vigilância sanitária não contempla a detecção de um possível risco nutricional para o paciente.

0 presente estudo ancorou-se no arcabouço conceitual enas abordagens da avaliação da qualidade de serviços de saúde indicados por Donabedian ${ }^{12}$, que metodologicamente propõe a avaliação de estrutura, processo e resultado. Destaca-se que uma boa estrutura pode condicionar, mas não determinar uma boa qualidade do serviço prestado, sendo, portanto, necessário introduzir componentes de avaliação de processo ede resultados para uma avaliação mais abrangente e consistente por parte da vigilância sanitária. 0 trabal ho teve por objetivo avaliar o cuidado nutricional em oito hospitais públicos, em quatro estados do Brasil, que receberam aportes do Pro- grama QualiSUS $S^{13}$ - programa voltado para a melhoria da qualidade da assistência hospitalar, a partir de uma reforma mais estrutural, cujo foco inicial eram os grandes hospitais.

\section{$M$ aterial e métodos}

Este estudo exploratório, transversal e descritivo, abrangeu oito hospitais públicos incluídos no QualiSUS até julho de 2005, nos quatro estados brasileiros contemplados pelo programa naquele momento: Pernambuco, Rio Grande do Sul, Sergipe e Rio de Janeiro. No Rio de Janeiro, primeira localidadea receber recursos de investimento em razão da crise do sistema de saúde, a pesquisa abrangeu a totalidade dos cinco hospitais do programa QualiSUS. N os demais estados, selecionou-se um hospital, e o critério de escolha baseou-sena importância deleparaa rede local de serviços de saúde. Os hospitais foram identificados numericamente, de um a oito. A pesquisa foi realizada no período de dezembro de2005 a fevereiro de 2006.

A pós revisão da legislação e dos instrumentos disponíveis para avaliar os serviços de saúde, elaboraram-se três instrumentos distintos para coleta de dados utilizando como base a legislação sanitária ${ }^{14-16}$, os M anuais de A creditação H ospitalar ${ }^{17,18} \mathrm{e} 0$ instrumento do Programa Nacional de Avaliação de Serviços de Saúde ${ }^{19}$, do exercício profissional ${ }^{20}$. Esses instrumentos, além de se basearem em normas e padrões estabelecidos e conhecidos, foram validados após pré-teste de resultado satisfatório, realizado em dois hospitais não incluídos na pesquisa, mas com indivíduos pertencentes a cada um dos grupos entrevistados. Em um dos questionários, utilizado para entrevistar o chefe do serviço de nutrição do hospital, constavam 39 perguntas, divididas em três seções: (1) recursos humanos; (2) manuais e procedimentos; (3) assistência nutricional. 0 segundo questionário, destinado ao responsável técnico pela unidade de alimentação e nutrição (UAN), continha 24 perguntas divididas em duas seções: (1) estrutura, manuais eprocedimentos; (2) assistência nutricional. 0 terceiro instrumento desenhado foi um check list para revisão de prontuários, com 13 itens de verificação. Esses instrumentos mantiveram as seções propostas nos documentos originais. A revisão de prontuários objetivou identificar a existência de dados registrados relacionados ao cuidado nutricional, privilegiando a presença da informação e não a compatibilidade com padrões. 
Em resumo, aplicaram-se questionários semiestruturados em 16 nutricionistas, dois em cada hospital, sendo oito chefes do serviço de nutrição do hospital e oito responsáveis técnicos pela unidade de alimentação e nutrição (UAN); observou-se diretamente 0 trabalho da equipe de nutrição nos locais em que eram desenvolvidas as atividades, segundo proposições contidas na Resolução do CFN no 380, de 200520; revisaram-se 219 prontuários abertos, que se encontravam nas enfermarias de clínica médica por pertencerem a pacientes internados nos hospitais públicos selecionados, variando de 25 a 30 prontuários por hospital.

A pesquisa foi submetida ao Comitê de Ética em Pesquisa da Escola Nacional de Saúde Pública Sergio Arouca, em cumprimento à Resolução $n^{\circ}$ 196/9621. Relatórios específicos, com as informações de seu interesse, foram encaminhados à direção de cada hospital participante da pesquisa.

\section{Resultados}

As entrevistas, bem como a observação direta, revelaram número insuficiente de profissionais de nutrição na equipe ${ }^{20} \mathrm{em}$ todos os hospitais pesquisados, o que é um obstáculo para a realização efetiva das atividades relacionadas ao cuidado nutricional por esse profissional. Em ne nhum dos hospitais estudados existia divisão do trabal ho do nutricionista - ou responsabilidade específica atribuída ao nutricionista - por leito ou por paciente. Existia, sim, a atribuição de responsabilidades por clínicas, independentemente do seu número de leitos. Calculou-se, a despeito disso, a quantidade de pacientes por profissional, e ela era sempre igual ou superior a 50, che gando a perfazer 150 pacientes.

Tabela 1. Realização referida pelos nutricionistas de componentes da assistência nutricional nos oito hospitais.

\begin{tabular}{lc}
\hline $\begin{array}{c}\text { Componentes da assistência } \\
\text { nutricional }\end{array}$ & $\begin{array}{c}\text { No de hospitais que } \\
\text { realizam as atividades }\end{array}$ \\
\hline Avaliação nutricional & 0 \\
Visita diária & 7 \\
Prescrição dietética & 1 \\
Evolução nutricional & 1 \\
Avaliação do índice de resto-ingestão & 0 \\
Pesquisa de opinião & 0 \\
Orientação ao paciente sobre a dieta & 0
\end{tabular}

Quanto aos principais componentes da assistência nutricional (Tabela 1), os profissionais denutrição entrevistados referiram, em todos os hospitais, não realizar avaliação nutricional na admissão dos pacientes, nem no decorrer da internação. Somente em um hospital o nutricionista realizava prescrição dietética e fazia evolução nutricional em prontuário. N os demais, afirmou-se que era o médico quem fazia a prescrição eo nutricionista, se necessário, fazia a alteração da prescrição em formulário próprio do setor de nutrição, ou seja, mantinha-se a prescrição original do médico inalterada. Com exceção de um dos hospitais, os nutricionistas afirmaram visitar diariamenteos pacientes, sendo a aceitação da dieta o principal item observado. Entretanto, em nenhum hospital existiam avaliação do índice de resto-ingestão ( parte da refeição não ingerida pelo paciente) eformulário para aferir 0 grau de satisfação dos pacientes em relação à alimentação. Quanto à orientação ao paciente sobre a sua dieta, em nenhum dos hospitais essa prática foi observada, mas todos os nutricionistas entrevistados afirmaram que esse procedimento era realizado na alta hospitalar através de impressos padronizados.

Em nenhum hospital os profissionais da equipe de saúde solicitavam formalmente parecer da nutrição. Em dois hospitais os profissionais da fonoaudiologia e do serviço social solicitavam parecer à nutrição, mas eram utilizados formulários próprios que não eram anexados ao prontuário. Todos relataram existir diálogo entre a equipe de enfermagem e a de nutrição sobre a aceitação da dieta.

Ao analisarmos os hospitais em relação à existência de Equipe Multidisciplinar de Terapia Nutricional (EMTN), verificou-se que três hospitais não possuíam essa equipe. Quanto aos hospitais que afirmaram possuir, quando solicitado, somente um deles demonstrou, através do livro de ata, a constituição formal da EMTN .

A revisão de prontuários revelou a ausência, em $93 \%$ dos prontuários, de registro, por qualquer profissional, sobre o estado nutricional na admissão dos pacientes. Também não se verificaram registros pela nutrição de prescrição dietética, avaliação nutricional, nem evolução nutricional dos pacientes, em sete hospitais. No único hospital em quese encontraram registros de prescrição e evolução nutricional por nutricionistas, $41,4 \%$ dos prontuários revisados não apresentavam esses registros.

Nos prontuários dos pacientes em terapia nutricional, não constavam registros sobre o es- 
tado nutricional. Um hospital possuía formulários próprios para avaliação nutricional e evolução dos pacientes em terapia nutricional, mas que não eram anexados ao prontuário dos pacientes. Em nenhum prontuário encontraram-se registros sobre a ocorrência de - ou fornecimento de orientação sobre - interação drogas versus nutrientes, reconhecidamente um dos padrões a serem alcançados na avaliação da qualidade do serviço ${ }^{18}$. Além disso, essas interações podem afetar o estado nutricional causando deficiências nutricionais, sendo a magnitude da interação dependente da natureza do medicamento e do estado nutricional do indivíduo ${ }^{22}$. Também não foram encontradas solicitações por escrito, por parte de qualquer profissional da equipe de saúde, de parecer nutricional, corroborando o járe latado na entrevista com os chefes da nutrição.

Em relação à existência de locais próprios, adequados e separados para o preparo de fórmulas lácteas e nutrição enteral, observou-seque apenas três hospitais estavam adequados. Nos demais, além de essas atividades serem desenvolvidas em um único setor, existiam problemas estruturais e fluxo inadequado. Em um dos hospitais foi identificada uma situação de risco sanitário, pois na única sala utilizada para o preparo defórmulas lácteas e dietas enteraistambém ocorria produção de dietas líquidas.

Oito unidades se encontravam com inadequações deestruturafísica esete no fluxo deprodução dealimentos ${ }^{14 \cdot 16,23}$. Ou seja, não eram cumpridos os processos que garantem um fluxo ordenado em todas as etapas da produção. Em relação aos aspectos gerais que envolvem a produção de refeições, verificou-se que nenhuma UAN possuía o M anual de boas práticas (M BP) atualizado, documentado e devidamente implantado. Todas as unidades necessitavam de adequação para que os recursos para lavagem das mãos estivessem disponíveis. Quanto às ações de educação para os profissionais envolvidos na produção de alimentos, foi informado que elas existiam, entretanto nenhum hospital apresentou programa, nem cronograma de real ização das ações, o que sugere que se essas ações são realizadas, não são feitas de modo sistemático. Também foi verificado que não havia participação da Comissão de Controle de Infecção H ospitalar no treinamento para os manipuladores de alimentos.

Em todos os hospitais, as copas funcionavam apenas como apoio para o porcionamento dealimentos ehigienização de recipientes; no entanto, não havia disponível sabão antisséptico ou qualquer outro material ou método para sa- nitização deutensílios eantissepsia das mãos dos manipuladores. A falta de padronização das porções também é problema comum nos hospitais, já que suas variações dependem do funcionário que serve a alimentação ${ }^{3}$, reforçando a importância da supervisão do fracionamento.

Todas as UAN possuíam um nutricionista como responsável técnico, e o serviço de produção de alimentos era realizado por empresa terceirizada em sete delas. Em todos os hospitais, os nutricionistas das UAN não trabal havam de forma integrada com a equipe de nutrição clínica, cabendo àqueles apenas gerenciar a produção de alimentos.

\section{Discussão}

A nutrição eo conjunto deatividades queela compreende constituem-se em uma parte importante da atenção oferecida ao paciente pelo hospital, fazendo parte do tratamento e da recuperação de seus pacientes ${ }^{24}$. Portanto, o hospital deve possuir um serviço de nutrição e dietética (SND) estruturado, organizado e integrado às outras áreas da atenção, tendo como função prestar assistência alimentar enutricional por meio da prescrição de dietas com atributos de qualidade e segurança do ponto de vista higiênico-sanitário, da orientação aos pacientes e, ainda, do monitoramento dos efeitos e aceitação da dieta. Dessa forma, o SND visa minimizar os riscos relacionados a uma dieta inadequada fornecida aos pacientes, em razão tanto de seu aspecto nutritivo quanto de contaminações inerentes ao ambiente hospitalar, protegendo-os de possíveis toxinfecções alimentares.

0 principal problema apontado pelos serviços de nutrição foi o número insuficiente de nutricionistas, percebido como o nó crítico para a qualidade do cuidado nutricional, o quetem sido indicado em outros trabalhos ${ }^{6,25}$. A alocação do nutricionista por clínicas, desconsiderando-se 0 número de pacientes e seu risco nutricional, na ausência de uma relação compatível entre o número de nutricionistas e 0 de pacientes com as atividades que necessitam ser desenvolvidas, favoreceuma fragilidade da assistência nutricional. E isso ocorre pela impossibilidade de se prestar assistência individualizada adequada ${ }^{26}$, no mínimo 12 horas/dia ininterruptas, inclusive nos fins de semana e feriados, conforme preconizado ${ }^{20}$.

Apesar de para todos os hospitais ter-se calculado que, na prática, os nutricionistas se responsabilizavam por 50 a 150 pacientes, não se constatou a existência de classificação do atendi- 
mento nutricional por níveis (primário, secundário eterciário), de acordo com os fatores de risco nutricional. A proporcional idade de pacientes por nutricionista é fixada por norma para os níveis primário, secundário eterciário, respectivamente em até 60,30 e 15 pacientes por nutricionista ${ }^{20}$.

As atribuições específicas do nutricionista em hospitais, entre outras, são avaliar o estado nutricional, estabelecer a dieta eregistrar diariamente no prontuário do paciente a prescrição dietoterápica, a evolução nutricional e as intercorrên$\operatorname{cias}^{20}$. No estudo de Boog e Silva ${ }^{27}$, a ausência do nutricionista para proceder à avaliação nutricional rotineira dos pacientes foi apontada pelos enfermeiros como um problema corrente. Os achados empíricos do estudo, relativos às atribuições específicas dos nutricionistas no processo de cuidado nutricional, mormente os referentes às suas ações de diagnóstico, prescrição ere gistro no prontuário, apontam a falta de correspondência entre algumas normas ${ }^{20} \mathrm{e}$ a realidade observada em sete dos oito hospitais da pesquisa, o que, em grande parte, é corroborado por outros autores $3,26,28$.

De modo semelhante, para Ulíbarri28, poucos hospitais conseguem se programar para a detecção precoce da desnutrição dos pacientes que ali ingressam, e menos ainda se organizam para acompanhar a evolução durante a internação. Segundo este mesmo autor, a desnutrição é detectada em estágios demasiadamente avançados, não se dispondo, rotineiramente, denenhumaferramenta útil para prevenção, detecção precoce, registro, seguimento econtrole da evolução nutricional depacientes. Na mesma linha, Garcia et al. ${ }^{10}$ ressaltam que a equipe de nutrição deve ocupar-se não apenas de fazer o diagnóstico nutricional, mas também de criar critérios deacompanhamento e controle dos fatores que contribuem para os problemas nutricionais.

Os dados sobre a prevalência e as condições agravantes da desnutrição hospitalar citados ${ }^{8-11}$ corroboram a necessidade de monitoramento do estado nutricional, tanto na admissão quanto na permanência hospitalar, bem como de planejamento, distribuição e avaliação da alimentação hospital ar, uma vez que a desnutrição tem como consequência elevação da mortalidade hospitalar e internações prolongadas gerando demanda reprimida, além de readmissão mais rápida com consequenteelevação dos custos ${ }^{29}$. Deacordo com Boog e Silva ${ }^{27}$, mais importante do que intervir na desnutrição hospitalar é preveni-la. Para isso, concorrem a avaliação do estado nutricional e o acompanhamento da evolução do paciente in- ternado, também no que concerne aos aspectos nutricionais.

Esperança ${ }^{30}$ avaliou percentuais de resto-ingestão em hospitais, público e privado, e verificou quea adequação calórica da alimentação efetivamente consumida pelos pacientes apresentou valor médio de $65,3 \%$ das necessidades, podendo assim comprometer a recuperação dos pacientes. Conforme referido pelos nutricionistas e demonstrado na Tabela 1, em nenhum dos hospitais da pesquisa se orienta o paciente quanto à sua dieta, nem se procede ao cálculo de indicadores sobre aceitação dela. Todavia, em sete hospitais foi referido que a equipe de nutrição realiza visita diária aos pacientes. E é por meio dessa visita diária que o nutricionista avalia a aceitação da dieta, informa-se sobre o motivo de uma possível recusa al imentar e realiza educação alimentar. A análise desses dados da Tabela 1 levanta uma interrogação sobre o objetivo e a efetividade dessa visita para educação nutricional.

Diferentemente do que foi referido na entrevista, notou-se no período da pesquisa a ausência do profissional de nutrição no momento do porcionamento das dietas, bem como na supervisão da distribuição das refeições aos pacientes. Além disso, não havia registros sobre parecer nutricional nos prontuários revisados, e os profissionais entrevistados referiram que o diálogo com os outros membros da equipe de assistência, à exceção da enfermagem, não ocorria regularmente. Esses fatores contrariam normas que apontam a integração do nutricionista na equipe multidisciplinar, com participação plena na atenção prestada ao paciente e atribuição específica na assistência hospitalar ${ }^{20}$.

Segundo o Regulamento Técnico para a Terapia de Nutrição Enteral, as unidades hospitalares que queiram habilitar-se à prática dessa terapia devem contar com a EMTN, grupo obrigatoriamente constituído de, pelo menos, um médico, nutricionista, enfermeiro e farmacêutico, com treinamento específico para essa atividade, sendo 0 ato formal de constituição da equipe um item imprescindível do rotei ro deinspeção ${ }^{16}$. Apesar da referência a essa comissão em cinco hospitais, apenas um cumpriu a exigência formal de ato de constituição.

O funcionamento da EMTN na maioria dos hospitais brasileiros se dá pelo estabelecimento de diretrizes gerais e protocolos de conduta nutricional a serem seguidos pela equipeassistencial que conduz o doente; em outros hospitais, a EM NT tem atuação clínica, avaliando diretamente os doentes mediante solicitação da equipe as- 
sistencial ${ }^{25}$. Apesar de um papel importante, são apontadas dificuldades na implantação dessa equipe, seja pela falta de informação e de recursos disponíveis, seja ainda por questões de política hospitalar e da pouca aceitação por parte da equipe assistencial ${ }^{25}$. Essa constatação reforça a dificuldade de trabal ho multidisciplinar no que concerne ao cuidado nutricional, que tem sido indicada nesta pesquisa.

Em relação às unidades de alimentação enutrição, não foi propósito deste estudo apontar exaustivamente as inadequações encontradas, mas algum comentário faz-se necessário. A inexistência do $M$ anual de boas práticas de manipulação (M BPM) que descreva as rotinas técnicas para garantir o controle higiênico-sanitário dos alimentos está em desacordo com a Portaria MS $n^{\circ} 1.428 / 93$, queaponta a necessi dade de queesse instrumento seja elaborado ${ }^{14}$. O M BPM necessita estar adequado às características e às atividades desenvolvidas em cada unidade e, obrigatoriamente, ser implementado.

Outros dois importantes aspectos em desacordo com a legislação sanitária vigente são: a não disponibilidade, em número suficiente, de locais adequados para higienização das mãos ${ }^{15}$; e a ausência de controle de treinamentos para manipuladores de alimentos nas unidades hospitalares. Vale sal ientar que uma das vias de contaminação hospitalar é a ingestão de alimentos contaminados, eos manipuladores de alimentos podem ser considerados agentes disseminadores de microrganismos no processo de manipulação ${ }^{31}$. Ao mesmo tempo, entre os aspectos técnicos que mais preocupam os funcionários da cozinha está a questão da contaminação ${ }^{3}$.

Ressalta-se a importância da participação da Comissão de Controle de Infecção Hospitalar $(\mathrm{CClH})$ no treinamento dos manipuladores de alimentos, o que não se verificou neste estudo. Isso constitui-se em um problema também para a $\mathrm{CCIH}$, na medida em que uma das vias de infecção hospitalar é a ingestão de alimentos contaminados, e que uma das causas dessas infecções pode ser a falta de um programa de treinamento de boas práticas de higiene para os indivíduos quetrabalham direta ou indiretamentecom pessoas internadas. Se há contaminação, a qualidade do alimento, resultado da combinação de atributos microbiológicos, nutricionais e sensoriai $\mathrm{s}^{32}$, está prejudicada.

Um estudo que tratou da gestão das atividades relacionadas à produção das refeições ${ }^{33}$ evidenciou fragmentação das atividades pela imprevisibilidade ligada ao processo de produção de refeições (matérias-primas, pessoas e estrutura), além da pressão temporal em dar respostas, diagnosticar e tomar decisões em curto prazo. Verificou-se que, em todos os hospitais, o trabalho da equipe de nutrição éfragmentado, com dicotomia entreprodução de dietas e assistência nutricional, sendo a precariedade estrutural e a baixa integração entre nutricionistas da clínica e da produção de alimentos os traços comuns entre os dois setores.

De acordo com Souza e Proença ${ }^{33}$, a gestão das atividades relacionadas à produção das refeições é uma ferramenta essencial para a garantia da qualidade do atendimento ao paciente. Por sua vez, essas atividades distanciam o profissional dos principais objetivos de uma unidade de alimentação e nutrição hospitalar, ou seja, da prevenção de agravos e recuperação do estado nutricional dos pacientes. Assim, o nutricionista que foi contratado para atender com prioridade os pacientes passa a se ocupar exclusivamente com atividades administrativas, ficando o paciente em segundo plano ${ }^{24}$.

No entanto, esse afastamento pode ocorrer por outros fatores fora do controle do nutricionista. Em primeiro lugar, pela inserção do serviço de nutrição e dietética (SND) na estrutura hospitalar como um serviço de apoio; pelo fato de a assistência nutricional ainda não ser uma prática instituída; pelo desempenho esperado institucionalmente de o SND ser a produção de refeições ${ }^{3}$. Também a inexistência de local adequado e próximo para o nutricionista realizar suas atividades de cunho administrativo ou técnico, seja na área de produção de refeições, seja nas enfermarias, lhe dificulta 0 acesso ao paciente e a integração de suas ações junto a outros ocupacionais ou profissionais ${ }^{3}$.

Os resultados do estudo sugerem fragilidade no cuidado nutricional hospitalar principalmente pelo profissional que, pela formação, detém 0 maior conhecimento dessa prática e que teria a centralidadeno processo. A baixa qualidadedesse cuidado pode agravar o risco nutricional, piorando o estado de saúde dos pacientes, bem como onerando o custo hospitalar.

A avaliação do processo de cuidado nutricional permitiu inferir que o principal entrave para a qualificação da assistência está relacionado à insuficiência de recursos humanos. Cabe ressaltar a importância de outros estudos para subsidiar medidas que possam contribuir para meIhor qualidade da assistência nutricional nos hospitais do país.

Os achados da revisão de prontuários salientaram a precariedade da dimensão multiprofis- 
sional do cuidado nutricional: a inexistência de registros, por qualquer profissional, em $93 \%$ dos prontuários, sobre o estado nutricional na admissão dos pacientes; o fato de não se encontrarem registros sobre o estado nutricional em pacientes em terapia nutricional. Ambos os achados são demonstrativos dessa precariedade e sugestivos da necessidade de revalorização da importância da alimentação no cuidado do paciente para a busca conjunta de soluções, mesmo quando 0 estado do paciente não exige grande intervenção ${ }^{6}$.

A vigilância sanitária, pelas suas prerrogativas, pode contribuir para a melhoria da qualidade do cuidado nutricional se incorporar padrões relativos a ele na sua avaliação. Ela tem um saber específico quanto à qualidade e segurança sanitária de produtos e serviços que precisa dialogar com os saberes dos profissionais que atuam nas demais ações de saúde ${ }^{34}$, bem como com a sociedade, considerando-se a necessidade desta última, no limite, de definir os riscos com os quais aceita conviver.

\section{Conclusões}

De modo geral, os resultados deste estudo apontam para uma baixa integração das atividades de assistência nutricional ao conjunto de ações desenvolvidas pela equipe de saúde no ambiente hospitalar. Esse fato pode ser respaldado pela observação da ausência de registros em prontuários e pela insuficiência de profissionais necessários para realização da avaliação e do acompanhamento nutricional, especialmente para pacientes sob risco nutricional. Todavia, elementos relacionados à organização do trabalho também parecem contribuir para certo déficit de qualidade. Dentreesses, a não classificação dos pacientes segundo a avaliação nutricional realizada próxima ao momento da admissão, seguida da priorização do atendimento individualizado eespecializado daqueles que se encontram em condição ou situação de maior risco.

É certo quea pesquisa tomou como uma importante fonte de dados o prontuário - e, no caso, apenas os prontuários em uso no momento da visita aos setores de clínica médica -, em que há reconhecidamente falta de registro sistemático das ações nutricionais (estado nutricional do paciente, consumo energético e de nutrientes, entreoutras) pela equipedenutriçãa ${ }^{3}$, mas também pelo conjunto de profissionais de saúde no que concerne aos aspectos nutricionais $s^{35,36}$. M as também foi observada a falta de nutricionistas, evidenciada pela ausência ou pela implementação deforma incipientedealguns dos componentes da assistência nutricional, demonstrando uma lacuna no processo de cuidado aos pacientes, mormente no caso daqueles em risco nutricional. Pacientes em terapia nutricional são extremamente vulneráveis, portanto o cuidado nutricional deve ser prestado ao paciente em sua plenitude, tanto para prevenir quanto para reverter quadros de desnutrição e outros agravos relacionados à nutrição.

O estudo não se propôs a avaliar ou recomendar a adoção de um dado método de avaliação, rastreamento da desnutrição ou de intervenção e avaliação da evolução do cuidado nutricional. Em primeiro lugar, pelo reconhecimento da existência de uma diversidade de métodos desenvolvidos para contextos de aten ção a grupos específicos de pacientes; em segundo, porque se assume que, sendo o aprimoramento de processos específicos, como os referentes ao cuidado nutricional, fundamental para se aprimorar a atenção prestada ao paciente internado, os processos de melhoria devem ter forte participação dos profissionais envolvidos no cuidado, que precisam chamar para si a responsabilidade da tomada de decisão e serem apoiados pela gestão da unidade hospitalar.

No entanto, reconhecendo quenão sepodem deixar os necessários processos de melhoria ao sabor das iniciativas e vontades, seja dos profissionais, seja do gestor hospitalar, no estudo se recomenda que a vigilância sanitária incorpore elementos da avaliação de processo e de resultado, bem como adote a revisão de prontuários durante a inspeção sanitária. Assim, espera-se que sua ação contribua para impactar positivamente os indicadores do estado nutricional dospacientes, e quesua própria prática também seaprimorecom a incorporação de outros indicadores e padrões, ultrapassando a verificação dos quesitos estruturais, importante e legítima, mas não suficiente. 


\section{Colaboradores}

MH DeSeta e G O'Dwyer orientaram a pesquisa e revisaram a versão final do artigo; P H enriques e GLP Sales coletaram dados e redigiram a primeira versão do artigo.

\section{Referências}

1. M achado FM S, Simões AN. Análise custo-efetividade e índice de qualidade da refeição aplicados à estratégia global da OMS. Rev Saude Publica 2008; 42(1):64-72.

2. Bryan FL. Hazard analysis critical control point HACCP: systems for retail food and restaurant operation. J Food Prot 1990; 53(11):978-983.

3. Garcia RWD. A dieta hospitalar na perspectiva dos sujeitos envolvidos em sua produção e em seu planejamento. Rev Nutri 2006; 19(2):129-144.

4. Hammond KA. Avaliação dietética e clínica. In: $M$ ahan LK, Stump SE, organizadores. Krause: alimentos, nutrição e dietoterapia. 10a ed. São Paulo: Roca; 2002. p. 341-366.

5. Brylinsky C. Processo de cuidado nutricional. In: $M$ ahan LK, Stump SE, organizadores. Krause: alimentos, nutrição e dietoterapia. 10a ed. São Paulo: Roca; 2002. p. 416-433.

6. Campos SH, Boog, MCF. Cuidado nutricional na visão de enfermeiras docentes. Rev Nutri 2006; 19(2):145-155.

7. Ferreira VF, Magalhães R. Nutrição e promoção da saúde: perspectivas atuais. Cad Saude Publica 2007; 23(7):1674-1681.

8. Waitzberg DL, Gama-Rodrigues J, Correia MI. Desnutrição hospitalar no Brasil. In: Waitzberg DL, organizador. Nutrição oral, enteral e parenteral na prática clínica. São Paulo: Atheneu; 2000. p. 385-397.

9. Waitzberg DL, Correia MI. Hospital malnutrition: the Brazilian National Survey (Ibranutri): a study of 4,000 patients. Nutrition 2001; 17:553-580.

10. Garcia RWD, Leandro-M erhi VA, Pereira AM. Estado nutricional e sua evolução em pacientes internados em clínica médica. Rev Bras Nutr Clin 2004; 19:59-63.

11. M ello ED, Beghetto MG, Teixeira LB, Luft VC. Desnutrição hospitalar cinco anos após o Ibranutri. Rev Bras Nutr Clin 2003; 18:65-69.

12. Donabedian A. The quality of care: how can it be assessed? JAM A 1988; 260(12):1743-1748.

13. Brasil. M inistério da Saúde. Secretaria Executiva. QualiSUS: Política de Qualificação de Atenção à Saúde. Documento de trabalho. Brasília, 15 jun 2004.

14. Brasil. M inistério da Saúde. Portaria no 1.428, de 26 de novembro de 1993. Aprova o Regulamento Técnico para inspeção sanitária de alimentos, as diretrizes para o estabelecimento de boas práticas de produção e de prestação de serviços na área de alimentos e o regulamento técnico para o estabelecimento do padrão de identidade e qualidade para serviços e produtos na área de alimentos. Diário O ficial da União 1993; 2 dez.

15. Brasil. M inistério da Saúde. Secretaria de Vigilância Sanitária. Portaria no 326, de 30 de julho de 1997. Aprova o Regulamento Técnico sobre condições higiênico-sanitárias e de boas práticas de fabricação para estabelecimentos produtores/industrializadores de alimentos. Diário Oficial da União 1997; $1^{\circ}$ ago. 
16. Brasil. M inistério da Saúde. Agência Nacional de Vigilância Sanitária. Resolução RDC $n^{0} 63$, de 6 de julho de 2000. Aprova o Regulamento Técnico para fixar os requisitos mínimos exigidos para Terapia de Nutrição Enteral. Diário Oficial da União 2000; 7 jul.

17. Brasil. M inistério da Saúde. Secretaria de Assistência à Saúde. M anual brasileiro de acreditação hospitalar: série A, n 117. Braślia: M inistério da Saúde; 2001.

18. Consórcio Brasileiro de Acreditação de Sistemas e Serviços de Saúde/Joint Commission International. $M$ anual internacional de padrões de acreditação hospitalar. Rio de Janeiro: CBA/U erj/Cepesc; 2003.

19. Brasil. Ministério da Saúde. Secretaria de Atenção a Saúde. Departamento de Regulação, Avaliação e Controle de Sistemas. Coordenação Geral de Regulação e Avaliação. Programa Nacional de Avaliação de Serviços de Saúde. Brasília: M inistério da Saúde; 2004

20. Conselho Federal de Nutricionistas. Resolução CFN no 380, de 28 de dezembro de 2005. Dispõe sobre a definição das áreas de atuação do nutricionista e suas atribuições, estabelece parâmetros numéricos de referência por área de atuação, e dá outras providências. Diário Oficial da U nião 2006; 10 jan.

21. Brasil. Ministério da Saúde. Conselho Nacional de Saúde. Resolução no 196, de 10 de outubro de 1996. Estabelece os requisitos para realização de pesquisa clínica de produtos para saúde utilizando seres humanos. Diário Oficial da União 1996; 16 out.

22. Reis NT. Interação entre drogas e nutrientes. Revista Nutrição em Pauta 1999; 39:38-42.

23. Brasil. Resolução RDC $n$ ㅇ 50, de 21 de fevereiro de 2002 (versão consolidada pela GGTES). Aprova 0 Regulamento Técnico destinado ao planejamento, programação, elaboração, avaliação e aprovação de projetos físicos de estabelecimentos assistenciais de saúde, em anexo a esta resolução, a ser observado em todo o território nacional. [acessado 2009 jan 6]. Disponível em: http://e-legis.anvisa.gov.br/leisref/ public/showAct.php?id=25622\&word=

24. Mezomo IB. Os serviços de alimentação: planejamento e administração. São Paulo: M anole; 2002.

25. Leite HP, Carvalho WB, Santana e M enezes JF. Atuação da equipe multidisciplinar na terapia nutricional de pacientes sob cuidados intensivos. Rev Nutri 2005; 18(6):777-784.

26. Fonseca PC. Estado nutricional e adequação da ingestão alimentar em pacientes submetidos a laparotomia [dissertação]. Belo H orizonte: Universidade Federal de M inas Gerais; 2006.
27. Boog M CF, Silva JB. Percepções de enfermeiras sobre o processo do cuidado nutricional. Rev Bras Nutr Clin 2001; 16:17-22.

28. Ulíbarri JI. La desnutricion hospitalaria. Nutr Hosp 2003; 18:109-112.

29. Moreira Jr. JC, Waitzberg DL. Consequências funcionais da desnutrição. In: Waitzberg DL, organizador. Nutrição oral, enteral e parenteral na prática clínica. São Paulo: Atheneu; 2000. p. 399-409.

30. Esperança LMB. Estudo comparativo do desperdício alimentar observado em hospitais público e privado. Cadernos (Centro Universitário São Camilo) 1999; 5:68-76.

31. Salles RK, Goulart R. Diagnóstico das condições higiênico-sanitárias e microbiológicas de lactários hospitalares. Rev Saude Publica 1997; 31:131-139.

32. Souza CL, Campos GD. Condições higiênico-sanitárias de uma dieta hospitalar. Rev Nutri 2003; 16(1):127134.

33. Souza AA, Proença RPC. Tecnologias de gestão dos cuidados nutricionais: recomendações para qualificação do atendimento nas unidades de alimentação e nutrição hospitalares. Rev Nutr 2004; 17:425-436.

34. O'D wyer G, Tavares M FL, De Seta M H. O desafio de operacionalizar as ações de vigilância sanitária no âmbito da promoção da saúde e no locus saúde da família. Interface (Botucatu) 2007; 11(23):467-484.

35. Kanashiro M. Avaliação nutricional de crianças internadas nos hospitais de Belo H orizonte [dissertação]. Belo Horizonte: Universidade Federal de M inas Gerais; 2006.

36. Kanashiro $M$, Péret Filho LA, Penna FJ, Fuji JB. Avaliação pondo-estatural e condições para esta aferição em crianças internadas em hospitais de Belo H orizonte. Revista M édica de M inas Gerais 2008; 18(3):153-159.

Artigo apresentado em 10/02/2009

Aprovado em 28/08/2009

Versão final apresentada em 16/06/2010 